\title{
Reformas de ida y vuelta en la gobernanza horizontal del bienestar: el caso de la colaboración público-privada en la intermediación laboral en la Comunidad Valenciana ${ }^{1}$
}

\author{
Round-trip reforms in the horizontal governance of welfare: \\ the case of public-private collaboration in labor intermediation \\ in the Valencian Region
}

\author{
Daniel Catalá-Pérez \\ Universitat Politècnica de València \\ dacapre@ade.upv.es
}

\begin{abstract}
NOTA BIOGRÁFICA
Daniel Catalá-Pérez es Graduado en Gestión y Administración Pública por la UPV, Máster en Gestión de Empresas, Productos y Servicios (UPV) y M. A. International Product and Service Management (Universidad de Ansbach). Ha sido becario del programa JAE-INTRO del CSIC y becario de posgrado en el CIS. Trabaja en la UPV, como investigador predoctoral FPU, en su tesis doctoral sobre colaboración públicoprivada en políticas de innovación. Además de investigar el área de la colaboración público-privada en diferentes ámbitos, ha publicado también algunos trabajos sobre evaluación de políticas públicas, área en la que es Experto Universitario por la Universidad de Sevilla.
\end{abstract}

Eloísa del Pino

Instituto de Políticas y Bienes Públicos-Consejo Superior de Investigaciones Científicas eloisa.delpino@csic.es

\section{NOTA BIOGRÁFICA}

Eloísa Del Pino es Científica Titular, Instituto de Bienes y Políticas Públicas (IPP), Consejo Superior de Investigaciones Científicas (CSIC). Doctora en Ciencia Política por la UCM y licenciada en C. Política y en Derecho. Ha sido profesora de varias universidades españolas e investigadora invitada en el CNRS de Burdeos, en la School of Social Policy and Social Research (Universidad de Kent), la School of Political Studies (Universidad de Ottawa) y la Universidad de Oxford, entre otros lugares. Dirigió el Observatorio de Calidad de los Servicios Públicos, en la Agencia Nacional de Evaluación de Políticas Públicas y Calidad de los Servicios. Sus áreas de especialización son las políticas sociales y el Estado de Bienestar, la reforma de la administración pública y las actitudes de los ciudadanos hacia el Estado y las políticas públicas.

\section{RESUMEN}

Las ideas de la Nueva Gestión Pública y los procesos de emulación de políticas europeas han extendido la colaboración público-privada como instrumento de gobernanza horizontal en la provisión de bienestar.

\footnotetext{
1 Este trabajo se ha realizado en el marco del proyecto de investigación CRIGOBBE (Los efectos de la crisis en la gobernanza del sistema de protección social en España: la perspectiva multinivel y del welfare-mix) financiado por el Instituto Nacional de Administración Pública INAP para el período 2016-2017, en su convocatoria permanente para la contratación de proyectos de investigación. Daniel Catalá-Pérez, a su vez, es beneficiario de una ayuda predoctoral de Formación de Profesorado Universitario (FPU 15/03926) del Ministerio de Educación, Cultura y Deporte del Gobierno de España. Los autores quieren agradecer al editor y los dos evaluadores que han revisado este artículo sus valiosos comentarios y sugerencias que han contribuido a mejorar la calidad del mismo.
} 
Sin embargo, la búsqueda de una mayor participación del sector privado en la gestión de determinados servicios públicos no está exenta de dificultades. Un caso claro es la reforma de la intermediación laboral llevada a cabo en España desde 2013, cuyo alcance final ha sido muy limitado debido a factores como la crisis, el contexto de gobierno multinivel y la ideología de los gobiernos. En este artículo se analiza el caso concreto de la Comunidad Valenciana para tratar de entender las dificultades de reforma de este programa público y contribuir a la literatura con nueva evidencia empírica sobre la importancia de los factores citados en el grado de éxito de las reformas que persiguen incrementar la colaboración pública-privada en el sector de las políticas sociales.

\title{
PALABRAS CLAVE
}

Estado de Bienestar; Políticas sociales; Políticas Activas de Empleo; Intermediación Laboral; Gobernanza; Colaboración Público-Privada.

\begin{abstract}
The ideas of the New Public Management and the emulation of European policies have extended publicprivate collaboration as a horizontal governance instrument in the provision of welfare. However, the search for greater participation of the private sector in the management of certain public services presents difficulties. A clear case is the reform of labor intermediation carried out in Spain since 2013, whose final scope has been very limited due to factors such as the crisis, the multilevel government context and the ideology of governments. This article analyzes the specific case of the Valencia Region to try to understand the difficulties of reform of this public program and contribute to the literature with new empirical evidence on the importance of the factors cited in the degree of success of the reforms that seek to increase publicprivate collaboration in the sector of social policies.
\end{abstract}

\section{KEYWORDS}

Welfare state; Social Policies; Active Employment Policies; Labor Intermediation; Governance; PublicPrivate Collaboration.

\section{SUMARIO}

I. LOS CAMBIOS EN LA GOBERNANZA PÚBLICO-PRIVADA EN LAS POLÍTICAS ACTIVAS DE EMPLEO. II. LOS FACTORES EXPLICATIVOS DE LAS REFORMAS EN LA GOBERNANZA PÚBLICO PRIVADA EN LAS POLIITICAS ACTIVAS DESEMPLEO. III. LAS REFORMAS EN LA INTERMEDIACIÓN LABORAL: LA CPP EN ESPAÑA. IV. EL ACUERDO MARCO EN LAS CCAA: CONFLICTO COMPETENCIAL Y CRÍTICAS SOBRE LOS INSTRUMENTOS DE PROVISIÓN PRIVADA. V. EL CASO DE LA COMUNIDAD VALENCIANA: UN CAMINO DE IDA Y VUELTA. VI. DISCUSIÓN Y CONCLUSIONES. BIBLIOGRAFÍA.

\section{LOS CAMBIOS EN LA GOBERNANZA PÚBLICO-PRIVADA EN LAS POLÍTICAS ACTIVAS DE EMPLEO}

A lo largo de las dos primeras décadas del siglo XXI, las políticas de lucha contra el desempleo de los países europeos han mostrado una tendencia convergente en la dirección de disminuir la generosidad de las prestaciones, de incrementar la condicionalidad de las mismas y de incluir mecanismos de activación basados en una mayor implicación del parado en el proceso de retorno al empleo (Clasen y Clegg 2011; Del-Pino y Ramos 2016; López-Santana 2015). Específicamente, el objetivo de estos mecanismos de activación es la eliminación de obstáculos para que los individuos participen en el mercado laboral mediante instrumentos como la formación, los servicios de intermediación entre los trabajadores y los potenciales empleadores u otros mecanismos que buscan facilitar la (re)inserción en la actividad laboral (Bonoli 2011; Clasen y Clegg 2011; Kenworthy 2010).

Este cambio de paradigma hacia la activación implica no solo reformas en el contenido de las políticas de mercado laboral sino también una transformación en el modo en que las políticas son gestionadas y gobernadas (Klenk 2018). En el caso de la intermediación se han producido numerosos cambios en su gestión (Van Berkel, De-Graaf, y Sirovátka 2011; De-la-Porte y Heins 2014; Graziano y Raué 2011; Heidenreich y 
Aurich-Beerheide 2014; López-Santana 2016; Weishaupt 2010). Países como Suecia, Alemania, Dinamar$\mathrm{ca}$, Reino Unido y Holanda han reforzado este programa mediante planes individualizados de inserción laboral y sus correspondientes mecanismos de seguimiento (Hoogemboom 2011; Martin 2015; Mathias 2017; Van-Berkel y Borghi 2008). Igualmente, se ha modificado la gobernanza del sistema y ahora empresas especializadas trabajan con los gobiernos mediante distintas fórmulas de colaboración público-privada (CPP) (véase Greve y Hodge 2013; y Klenk 2018 sobre la CPP como un nuevo modelo de gobernanza). Es posible encontrar modelos de cuasi-mercado como el australiano, modelos basados en procesos de licitación concurrente entre proveedores y administración (Reino Unido u Holanda) y modelos mixtos, como el alemán, en los que la competencia y los factores de mercantilización se han trasladado a la relación entre el clienteconsumidor y los proveedores de servicios (Eurofound 2015; PwC-Asempleo 2014).

La introducción de estos mecanismos de CPP no está siendo sencilla e incluso en algunos países se ha llegado a revertir el proceso (Van Berkel et al. 2011; Breidahl y Larsen 2015). En España, tampoco está siendo pacífica (Jansen 2017). En este país, a partir de 2010, se inició un proceso de cambio de la gobernanza horizontal del sistema de intermediación laboral consistente en la puesta en marcha o expansión de la CPP siguiendo el modelo basado en procesos de licitación (Calvo-Gallego y Rodríguez-Piñero 2010; De-la-Rica 2015; De-Vicente Pachés 2012, 2013; Rodríguez-Piñero 2014). En particular, el caso de la reforma de la intermediación laboral en la Comunidad Valenciana está siendo llamativamente complejo pues los cambios muestran un camino de ida y vuelta. En 2013, el gobierno del PP (Partido Popular) en esta comunidad apostó por incorporar empresas privadas para la provisión del servicio de intermediación, en los términos legales planteados por el Gobierno Central, también del PP, al adherirse al Acuerdo Marco del que se hablará más adelante. Sin embargo, en 2015, con la llegada al Gobierno Autonómico de una coalición de izquierda formada por el Partido Socialista Obrero Español (PSOE) y Compromís, este gobierno se desvinculó del Acuerdo Marco, poco antes de que finalizara la licitación del que hubiera sido el primer contrato de colaboración con agencias de colocación en el ámbito autonómico valenciano.

En este artículo nos preguntamos cómo ha cambiado la gobernanza de las políticas activas de empleo en el caso de España y qué factores pueden explicar las decisiones de ida y vuelta adoptadas en la Comunidad Valenciana acerca de la implementación de este instrumento que permite participar a las empresas privadas en la gestión de las políticas activas de empleo. Para responder a tales preguntas analizamos la trayectoria del programa de intermediación laboral en los últimos años en España y, en particular, el caso de la Comunidad Valenciana, que es de máximo interés puesto que en un breve espacio de tiempo, se han producido dos cambios en relación con la gobernanza del sistema.

Dado que la reforma en el caso valenciano parece presentar algunas dificultades de consolidación debido a factores políticos, para entenderlas puede ser útil la literatura científica sobre los determinantes políticos que afectan a la reforma de las políticas sociales (The Politics of Welfare State Reform). Esta literatura sugiere que los procesos de cambio y el propio alcance de las reformas surgen de la interacción entre las ideas existentes en un contexto determinado, las instituciones y los actores (Bridgen y Meyer 2008; Gingrich 2011; Greve 2015; Pierson 2001:véase; Rothgang et al. 2008; Seeleib-Kaiser 2008). En este caso, las ideas sobre las políticas activas de empleo en el marco de la UE y según los expertos nacionales, así como sobre los paradigmas de gestión pública y la interacción de los actores en el sistema de gobierno multinivel parecen haber afectado a la decisión de transformar el modelo existente.

Desde un punto de vista metodológico, hemos analizado un solo caso mediante process tracing, metodología adecuada cuando deseamos saber cuáles son las relaciones entre las variables analizadas y si determinadas variables son suficientes para explicar el resultado de un proceso. En cuanto a las técnicas, hemos estudiado distintas fuentes documentales, textos legales, textos producidos por los servicios públicos de empleo, por entidades representativas del sector privado y por las propias agencias de colocación, además de informes de expertos. También hemos llevado a cabo diez entrevistas con diferentes actores implicados en el servicio de intermediación laboral en la Comunidad Valenciana, entre los que se encuentran directivos y técnicos de este mismo organismo, con gerentes de agencias de colocación y dirigentes de asociaciones estatales de estas mismas entidades. Es importante manifestar la dificultad encontrada para poder llevar a cabo entrevistas con personal del Servei Valencià d'Ocupació i Formació (Servef). Algunas de las personas entrevistadas solicitaron discreción sobre su participación en las entrevistas. Por ello hemos decidido no mencionar el puesto del entrevistado ya que fácilmente se identificaría a su ocupante. Estas entrevistas semiestructuradas se realizaron entre octubre de 2016 y enero de 2017 y en ellas se preguntó por las motivos que llevaron a emprender los cambios en del servicio de intermediación laboral por parte de la Generalitat y las dificultades de su puesta en marcha. De las diez entrevistas, ocho se realizaron 
de forma presencial y dos por vía telefónica. Únicamente tres entrevistados dieron su consentimiento para que la entrevista fuera registrada digitalmente, mientras que en el resto de los casos se tomaron notas manuscritas. Se recurrió a un análisis del discurso basado en diferentes niveles de lectura (intuitiva, temática, contextual y relacional).

Este artículo se organiza en cinco secciones. En la siguiente se realiza un breve repaso de los factores que explican la dirección y el alcance de los cambios en la gobernanza de las políticas sociales y se explican las principales hipótesis. En el tercer apartado se presta atención a la descripción del marco competencial, las reformas introducidas por el Gobierno Central a partir de 2010 y los motivos de las mismas. En la cuarta sección se aborda la implementación de las reformas en las CCAA en general y en Valencia en particular. En el quinto apartado se analiza la situación en que se encuentra el programa en la Comunidad Valenciana tras el cambio de gobierno regional y su desvinculación de la estrategia estatal. Finalmente, se plantean algunas conclusiones.

\section{LOS FACTORES EXPLICATIVOS DE LAS REFORMAS EN LA GOBERNANZA PÚBLICO PRIVADA EN LAS POLÍTICAS ACTIVAS DESEMPLEO}

Tres factores parecen influir en la decisión de reforma de la gobernanza de los sistemas de protección social: el papel de las ideas en un contexto socioeconómico concreto, la ideología de los gobiernos y otros actores sociales y las variables institucionales (Bridgen y Meyer 2008; Gingrich 2011; Greve 2015; Rothgang et al. 2008; Seeleib-Kaiser 2008).

La procedencia de la filosofía de la activación se suele atribuir al que algunos consideran como el nuevo paradigma de política social en los países europeos, el Estado orientado a la Inversión Social. La implicación de otros actores no-públicos en la prestación de servicios de titularidad pública no es ajena al funcionamiento de los Estados de Bienestar europeos desde su surgimiento. Sin embargo, debe reconocerse que las ideas de la Nueva Gestión Pública (NGP) y distintos procesos de emulación de políticas en el marco de la UE han extendido la CPP como un instrumento habitualmente utilizado en la administración del bienestar (Colino y Del Pino 2015; Pollitt y Bouckaert 2011). Aun así, estos procesos de marketization de las políticas de intermediación laboral no son siempre definitivos y encontramos algunos casos en que han sufrido reformas que podrían denominarse correctoras (por ejemplo, para evitar los efectos parking -no invertir recursos en los parados más difíciles- o creaming - priorizar una unos parados sobre otros- o mejorar la accountability) y otras que directamente han revertido este mecanismo de gobernanza de lo social (Breidahl y Larsen 2015; Klenk 2018).

Existe un debate ya clásico sobre la ideología de los gobiernos como uno de los factores que condiciona tanto el alcance de las políticas sociales como el tipo de instrumentos a través de los que éstas se implementan. En general, los defensores de los mecanismos de mercado, usualmente la derecha política, consideran que proporcionan una oportunidad para modernizar el Estado de Bienestar, ampliar o diversificar su oferta y calidad y disminuir sus costes. Todo ello en un contexto económico que podría ser calificado como de austeridad permanente (Pierson 2001), en el que los recursos de los gobiernos son limitados para satisfacer las crecientes demandas de la ciudadanía. Sin embargo, para su críticos, generalmente en la izquierda, muchos de estos instrumentos suponen la pérdida de control por parte del Estado de programas que tienen una gestión compleja, sin que esté claro si son más eficaces y eficientes o si pueden garantizar la misma cobertura y calidad de los servicios para todos los ciudadanos independientemente de su origen social (Ascoli y Ranci 2002; Klenk y Pavolini 2015; Seeleib-Kaiser 2008). Para este grupo, que con frecuencia posee una fuerte capacidad de movilización de los profesionales y ciudadanos, la situación se agrava aún más en contextos de crisis, cuando el papel de los servicios públicos es más necesario (Seeleib-Kaiser 2008).

Gingrich (2011) y Krumm (2016) han encontrado que todos los gobiernos, independiente de su color, recurren a instrumentos de CPP para la prestación de servicios, pero la primera autora también demuestra que la ideología determina el tipo concreto de instrumento y las justificaciones que los gobiernos esgrimen para usarlos. Bortolotti et al. (2004) encuentran que los gobiernos de derechas tienen a utilizar más estos instrumentos y a comenzar antes estos procesos, mientras que los de izquierdas son más proclives a la producción propia según Plantinga et al. (2011).

Otro factor que dificulta la puesta en marcha de las reformas de las políticas sociales y en particular de apertura de la provisión de los servicios del bienestar a actores privados, es que en ellos están implicados 
varios niveles de gobierno (Kazepov 2010; Oosterlynck et al. 2013; Hernández y Ramos 2017). En general, la literatura prevé que estos procesos sean más sencillos cuando los gobiernos implicados comparten el mismo color (Greer 2010). Las políticas activas de empleo son una de las áreas en la que con más frecuencia se han producido procesos de descentralización a otros niveles de gobierno en las últimas décadas (Bonoli 2011; Graziano 2012). Sin embargo, el alcance de la descentralización en esta política puede ser muy distinto según los países y, por lo tanto, la capacidad del Estado central de intervenir a través de incentivos o mecanismos regulatorios o financieros, por ejemplo, puede ser más o menos amplia. Igualmente puede variar la capacidad de los gobiernos subcentrales para diseñar sus propias políticas y resistirse a las pretensiones del Estado, en relación con la fórmula de provisión de los servicios (López-Santana 2015; Obinger y Starke 2014).

En cuanto a nuestras hipótesis, las ideas en el marco tanto del llamado social investment como de la NGP en el contexto de la UE deberían ayudarnos a explicar la CPP en el caso de la Comunidad Valenciana. Sin embargo, tales ideas no son suficientes para explicar lo que hemos denominado reformas de ida y vuelta y necesitamos recurrir también a la ideología de los gobiernos en un contexto multinivel.

\section{LAS REFORMAS EN LA INTERMEDIACIÓN LABORAL: LA CPP EN ESPAÑA}

El actual Sistema Nacional de Empleo español (SNE), se configura a partir de la aprobación de la Ley 56/2003 de 16 de diciembre, de Empleo, que regulariza el escenario provocado por el proceso de transferencia a las CCAA de las competencias en materia de políticas activas de empleo, iniciado en 1997 y culminado con la creación del servicio vasco de empleo en 2010. EI SNE está integrado actualmente por el Servicio Público de Empleo Estatal (SEPE), organismo autónomo adscrito al Ministerio de Empleo y Seguridad Social al que se le encomienda la gestión y el control de las prestaciones por desempleo (las llamadas políticas pasivas) y los 17 servicios públicos de empleo (SPE) autonómicos, encargados de gestionar los programas de empleo relativos a la formación profesional, la intermediación entre oferentes y demandantes en el mercado de trabajo y la orientación laboral.

EI SEPE planifica e impulsa propuestas de políticas de empleo en coordinación con los servicios autonómicos (Hernández-Moreno y Ramos 2017). Además, es habitual que las administraciones autonómicas presten los servicios de su competencia en colaboración con la administración local, con los agentes sociales o incluso con el sector privado, ampliando así el número de agentes que intervienen en el mercado de trabajo. Las organizaciones sindicales, por ejemplo, se implican generalmente en el desarrollo de iniciativas relacionadas con la formación y la orientación laboral de las personas desempleadas, del mismo modo que, cada vez más, existe un papel importante de empresas especializadas en este ámbito. Además de la colaboración entre los servicios públicos de empleo y el sector privado en la formación, el uso de mecanismos de gestión con la participación de actores del ámbito privado en servicios como el de la intermediación, es un fenómeno en expansión en España en los últimos años.

Hasta los años noventa, el modelo español de intermediación laboral se caracterizó por el monopolio estatal de un servicio de empleo público y gratuito, en el que la iniciativa privada no desempeñaba ningún papel, el uso de los servicios del antiguo Instituto Nacional de Empleo (INEM) era obligatorio y las políticas de empleo se diseñaban de forma centralizada. Con el PSOE en el Gobierno se produce la ruptura del monopolio del INEM a partir del Real Decreto-Ley 18/1993 de medidas urgentes de fomento de la ocupación. En la nueva situación, se mantiene un claro protagonismo de los operadores públicos, tanto en la prestación de servicios como en el control del funcionamiento del mercado de trabajo y de sus actores, pero a la vez, se abre la puerta a la iniciativa privada, limitada al trabajo temporal y a la colocación no lucrativa, uno y otra, sujetas a autorización administrativa (Rodríguez-Piñero 2014).

A partir de la evolución de aquel modelo se construye el modelo vigente, ajustando algunos elementos por medio de cambios normativos experimentados durante las reformas estimuladas en gran medida por la crisis económica y fiscal. Estas reformas se iniciaron en España con la Ley 35/2010, de medidas urgentes para la reforma del mercado de trabajo, aprobada durante el gobierno del PSOE, y continuaron con la Ley $3 / 2012$, de medidas urgentes para la reforma del mercado laboral, aprobada ya con el PP en el poder, que liberalizaron el servicio de intermediación laboral al reconocerse legalmente la capacidad de las agencias de colocación de carácter privado y de las empresas de trabajo temporal (ETT) de prestarlo en colaboración o no con los servicios públicos (Calvo-Gallego 2011; Calvo-Gallego y Rodríguez-Piñero 2010; De-Vicente Pachés 2012, 2013). En 2013, el gobierno del PP también aprobó el Acuerdo Marco para la selección de 
agencias de colocación para la colaboración con los Servicios Públicos de Empleo en la inserción en el mercado laboral de personas desempleadas al que se adhirieron 14 de las 17 CCAA y que articulaba un marco formal para la CPP entre las empresas del sector y los servicios públicos de empleo como fórmula de gobernanza (Rodríguez-Piñero 2014).

La situación creada por todas estas reformas, ha suscitado un intenso debate entre quienes defienden la gestión directa de este servicio y quienes ven en la provisión privada el remedio óptimo para mejorar el pobre desempeño de los SPE en esta materia (Alujas-Ruiz 2008; Consejo Económico y Social 2014; Fundación Élogos 2012; Jansen 2017; Ranstad Research 2015; Sánchez-Rodas Navarro 2014). Cabe tener en cuenta, que a través del servicio de intermediación laboral se implementan numerosos programas de (re) inserción laboral de colectivos desfavorecidos y se trata por ello de un servicio con un fuerte componente social y especialmente sensible a las medidas que puedan afectar a su prestación (Calvo y Lerma 2009).

Los defensores del carácter público de la intermediación laboral y de su prestación directa a través de los SPE se muestran en contra de lo que consideran en muchos casos la mercantilización o privatización del servicio (CCOO 2016; Molina-Navarrete 2012). Además, denuncian ciertos casos de mala praxis por parte de algunas agencias colaboradoras en determinadas CCAA (ADLYPSE 2015; Negueruela 2015, 2016). Por ello, demandan la reversión de algunas de las medidas adoptadas a lo largo de la última década, un mayor esfuerzo presupuestario de los gobiernos para aumentar los recursos humanos y técnicos contribuyendo así a la modernización de los servicios públicos de empleo, al tiempo que proponen mejorar la coordinación de los diferentes niveles de administración entre ellos y con otras entidades públicas o del Tercer Sector (Calvo y Lerma 2009; Nieto-Rojas 2012; Parreño 2015; Sánchez-Rodas 2014).

Los defensores de la colaboración con el sector privado son conscientes de los riesgos que este modelo de gestión conlleva. Dependiendo del diseño de los contratos de colaboración, las agencias privadas pueden tener incentivos para centrar sus esfuerzos en los trabajadores mejor posicionados (efecto cherry-picking o creaming) en detrimento de los más desfavorecidos (efecto parking) o incluso pueden rotar a sus clientes en contratos de corta duración para aumentar su remuneración (Bentolilla y Jansen 2013). Tampoco obvian la necesidad de favorecer la colaboración entre el sector público y otros agentes públicos o no lucrativos (Bentolilla y Jansen 2013; Fundación Élogos 2012), pero mantienen que se debe aprovechar la experiencia de las empresas especializadas (agencias de colocación y ETT). Argumentan que estas empresas conocen bien el mercado de trabajo y de las necesidades tanto de los empleadores como de los demandantes de empleo y ello, junto con una mayor flexibilidad, las coloca en posición de poder conseguir buenos resultados y ofrecer un servicio de calidad (Consejo Económico y Social 2014; Jansen 2016, 2017; Mullor 2011). Los argumentos de los defensores de la provisión privada se basan generalmente en el análisis de la situación en varios países que llevan aplicando esta fórmula con éxito desde hace años (Bentolilla y Jansen 2013; Jansen 2016; Mercader-Uguina 2014; Mullor 2011; PwC-Asempleo 2014; Ranstad Research 2015).

En el marco de este debate, podría decirse que existe un acuerdo extendido sobre los bajos niveles de eficacia y eficiencia de los SPE en intermediación laboral y una incidencia más bien escasa en el ámbito de la colocación, sobre todo a partir del año 1999 y especialmente en la actualidad (Alujas 2008; MercaderUguina 2014) ${ }^{2}$. Existe incluso cierto consenso en las causas que explican este modesto rendimiento (la escasez de recursos humanos y técnicos, la falta de confianza de los empresarios, la falta de adecuación entre la oferta y la demanda o la desconexión entre los niveles estatal y autonómico del SNE). Sin embargo, como se ha visto, las discrepancias son mucho mayores en relación a las soluciones que se proponen para resolver este problema (Alujas 2008; Fernández y Martín 2014).

Por otra parte, también la UE ha recomendado a las autoridades españolas que reformen el SNE y se ha instado a España a utilizar la CPP como instrumento de mejora de las políticas activas de empleo en lo referente a la colocación de desempleados. Por ejemplo, el ex comisario europeo de empleo, László Andor, pedía al Gobierno español un esfuerzo por ayudar a los desempleados a encontrar puestos de trabajo aumentando la capacidad de los servicios públicos de empleo y haciendo uso de las agencias de colocación

2 Este tema ha sido tratado también en prensa:

- ALCELAY, S. 2012. "Las agencias privadas buscarán empleo a los parados ante el fracaso del INEM". ABC. [27-12-2016] (http://www.abc.es/20120207/economia/abcp-agencias-privadas-buscaran-empleo-20120207.html).

- ALCELAY, S. 2013. "Solo 2,6 de cada cien nuevos empleos se consiguen a través del INEM". ABC. [28-12-2016] (http://www. abc.es/economia/20131209/abci-solo-cada-cien-nuevos-201312091222.html).

- ALCELAY, S. 2016. "Solo 1,9 de cada 100 nuevos empleos se logran a través del antiguo INEM". ABC. [28-12-2016] (http:// www.abc.es/economia/abci-solo-19-cada-100-nuevo-empleos-logran-traves-antiguo-inem-201606130138_noticia.html). 
GAPP. Nueva Época - N.19, mayo 2018 - ISSN: 1989-8991 - DOI: 10.24965/gapp.v0i19.10434 - [Págs. 107-122]

Reformas de ida y vuelta en la gobernanza horizontal del bienestar: el caso de la colaboración público-privada en la...

Daniel Catalá Pérez / Eloísa del Pino

privadas $^{3}$. También desde la OCDE, se ha instado al Gobierno a invertir en políticas activas desde un enfoque global que involucre al Gobierno y a las agencias de empleo ${ }^{4}$.

\section{EL ACUERDO MARCO EN LAS CCAA: CONFLICTO COMPETENCIAL Y CRÍTICAS SOBRE LOS INSTRUMENTOS DE PROVISIÓN PRIVADA}

La fórmula establecida para la colaboración entre las agencias de colocación privadas y los SPE en materia de intermediación laboral dentro del SNE, se construyó en torno a un Acuerdo Marco, aprobado en agosto de 2013 por la Dirección General del SEPE. El objetivo de este Acuerdo era preseleccionar un mínimo de 60 agencias de colocación (finalmente fueron 80 ) habilitadas para colaborar con los servicios públicos de empleo durante un máximo de cuatro años (dos años más dos posibles prórrogas anuales) y fijar las condiciones a las que se debían ajustar los posteriores contratos de colaboración entre las agencias seleccionadas y el propio SEPE o los servicios de empleo autonómicos que previamente se hubieran adherido voluntariamente a este Acuerdo Marco a través de un convenio con el SEPE. La adhesión al Acuerdo por parte de una comunidad autónoma suponía la aceptación de las condiciones de naturaleza económica, técnica y funcional a las que, según dicho acuerdo, debían ajustarse todos los contratos derivados del mismo. Inicialmente se adhirieron al Acuerdo todas las CCAA a excepción de Cataluña, País Vasco y Andalucía. Se estableció así un modelo en el que existía la posibilidad de que las agencias de colocación seleccionadas operaran en todo el país, a través de contratos con el SEPE o únicamente en una determinada comunidad autónoma, a través del contrato con el correspondiente servicio de empleo autonómico.

Con el Acuerdo Marco aprobado se inició el procedimiento de selección de las que serían las agencias de colocación colaboradoras. Este procedimiento sufrió importantes demoras debido a los recursos interpuestos por algunas de las agencias participantes, al considerar irregular la exigencia del cumplimiento individual de ciertos requisitos de solvencia financiera a agencias que se habían presentado formando uniones temporales de empresas (UTE), lo que podía dejarlas fuera del procedimiento. Finalmente, el Tribunal Administrativo Central de Recursos Contractuales resolvió que debía procederse a la acumulación de la solvencia financiera de cada una de las empresas que formaban las UTE, valorando la suma de las mismas. El proceso de selección concluyó casi un año después de la aprobación del Acuerdo Marco, en junio de 2014.

En ese momento, seleccionadas ya las agencias de colocación colaboradoras, entre las que se encontraban grandes ETT y pequeñas agencias de ámbito local o regional unidas en UTE, el SEPE inició la adjudicación de un primer contrato a nivel estatal entre el Ministerio y las agencias para colocar al colectivo de los desempleados del Plan Prepara ${ }^{5}$. El procedimiento de licitación de este primer contrato finalizó avanzado ya 2015 (la formalización del contrato tuvo lugar en agosto) después de que el Ministerio modificara la adjudicación original del contrato a las grandes ETT, y decidiera dejar la gestión de la intermediación laboral de este colectivo con especiales dificultades para encontrar empleo (el Plan Prepara incluye sobre todo a parados mayores de 45 años) en manos de pequeñas firmas locales, asociadas bajo la fórmula de UTE, más conocedoras y próximas, en principio, a sus necesidades concretas ${ }^{6}$.

A finales de mayo de 2015, aun sin haberse formalizado el primer contrato, el Gobierno aprobaba el inicio de licitación de un segundo contrato estatal entre el SEPE y las agencias de colocación. En este caso, la formalización fue mucho más rápida, se completó en septiembre de 2015 (un mes después del primer contrato) y el objeto del contrato era la colocación de demandantes de empleo en movilidad geográfica por todo el territorio nacional, diferentes de los incluidos en el Plan Prepara (Ministerio de Empleo y Seguridad Social 2015). Las agencias adjudicatarias, en este caso, sí fueron las grandes ETT con presencia en todo el país.

\footnotetext{
3 EUROPA PRESS. 2012. "Bruselas pide a España que refuerce el uso de agencias de colocación privadas para combatir el paro". europapress.es. [23-12-2016] (http://www.europapress.es/internacional/noticia-ue-bruselas-pide-espana-refuerce-uso-agenciascolocacion-privadas-combatir-paro-20120127174736.html).

${ }^{4}$ AGENCIA EFE. 2015a. "La OCDE advierte del bajo gasto en políticas activas de empleo de España". La Vanguardia. [29-12-2016] (http://www.lavanguardia.com/economia/20150922/54435415270/la-ocde-advierte-del-bajo-gasto-en-politicas-activas-de-empleo-de-espana.html).

5 MUNERA, I. 2014. "Hasta tres mil euros por cada parado de larga duración colocado". El Mundo. [28-12-2016] (http://www. elmundo.es/economia/2014/11/02/5453dbb1268e3e8a018b4579.html).

6 ZARAGOZÁ, J. L. 2015. "Las agencias privadas de empleo colocarán a solo mil de los 68.000 parados sin recursos". LevanteEMV. [28-12-2016] (http://www.levante-emv.com/economia/2015/03/10/agencias-privadas-empleo-colocaran-mil/1236359.html).
} 
Cabe recordar que la gestión de la intermediación laboral es una competencia autonómica y que el SEPE, en virtud de los dos contratos firmados con las agencias de colocación derivados del Acuerdo Marco de 2013, ha estado prestando este servicio en colaboración con las mencionadas agencias en todo el ámbito nacional. Si bien es cierto que inicialmente las CCAA que se adhirieron al citado Acuerdo Marco aceptaban las condiciones del mismo y de los contratos derivados, las tres comunidades no adheridas podrían haber visto invadidas sus competencias. Por ello, el gobierno del País Vasco, que junto a Cataluña han impulsado experiencias de CPP a través de su propio marco de actuación, recurrió el segundo de los concursos del SEPE ante el Juzgado central de lo contencioso-administrativo. Como medida cautelar, este órgano detuvo el proceso en el País Vasco. En enero de 2017, la sentencia dictada por este tribunal anuló exclusivamente en el territorio del País Vasco la contratación de las agencias de colocación colaboradoras por parte del SEPE debido a dicha invasión de competencias ${ }^{7}$.

Por otra parte, varias CCAA, tras las elecciones autonómicas de mayo 2015 decidieron desvincularse del Acuerdo Marco una vez transcurridos los dos primeros años de vigencia del mismo, momento en el cual podían optar por la renovación del convenio de adhesión al mismo o la desvinculación. Nos parece importante señalar que esta circunstancia se produjo en el caso de la Comunidad Valenciana, Castilla-La Mancha, Extremadura, Aragón y Baleares. En todas estas comunidades cambió el signo político de sus gobiernos pasando a gobernar el PSOE u otras coaliciones de izquierdas. El conflicto competencial fue uno de los diversos motivos argumentados para la desvinculación pero, en cualquier caso, según recoge el articulado del propio Acuerdo, «los contratos basados en el mismo subsistirán por su período propio de vigencia aun cuando el Acuerdo Marco se extinguiese». Es por ello que, tal y como veremos en la Comunidad Valenciana, en ciertas CCAA aun no estando adheridas al acuerdo el SEPE, se continuó prestando el servicio de intermediación laboral en colaboración con las agencias de colocación.

Además del aspecto competencial, el diseño del Acuerdo Marco levantó ciertas críticas en cuanto a los mecanismos de control y evaluación que establece para que la administración pública pueda asegurarse de que se presta un servicio eficaz y sobre todo eficiente. El Acuerdo deja cierta libertad a las CCAA a la hora llevar a cabo sus procesos de licitación con las agencias preseleccionadas, pero los criterios de selección no siempre son transparentes ni es se basen en la concurrencia competitiva. Además, a pesar de que el Acuerdo Marco fija una serie de criterios para la acreditación de la solvencia financiera y técnica, como se ha explicado en apartados anteriores, fueron numerosos los problemas referidos a las cuestiones relacionadas con los mismos, tanto en la selección de las agencias colaboradoras, como en el primer proceso de licitación planteado por el SEPE. Otro de los aspectos controvertidos del Acuerdo es la posibilidad de que las agencias de colocación puedan recibir una contraprestación económica cuando, fruto de su colaboración, un desempleado es sancionado, por ejemplo, por no acudir a una cita o rechazar una oferta de empleo adecuada (Negueruela 2015).

En esta misma línea del control y la eficiencia de la CPP, CCOO (2016) critica que el SEPE hiciera efectiva la prórroga de dos años que se establece en el Acuerdo Marco, sin haber llevado a cabo ninguna medida de seguimiento y evaluación de los resultados de la CPP, recordando que sólo se disponía de un informe provisional de agosto de 2016, publicado en ciertos medios ${ }^{8}$ y que no llegó a los agentes sociales hasta el mes de octubre, a pesar de las reiteradas peticiones de información. El sindicato denuncia que no es un informe detallado, ya que más allá de las cifras globales de personas atendidas o colocadas, no incluía información mínima sobre las características de los usuarios, actuaciones realizadas, resultados obtenidos, porcentaje de inserción, características de los contratos y ejecución presupuestaria detallada, por lo que no era útil para evaluar realmente la CPP y mejorar, en su caso, el contenido. Otro aspecto de los señalados por $\mathrm{CCOO}$, es que el Acuerdo Marco contempla un instrumento (la Comisión de Seguimiento) responsable de la dirección, inspección y control del Acuerdo, que transcurridos dos años desde la selección de las agencias privadas adjudicatarias del Acuerdo Marco y su puesta en marcha, tan sólo se había reunido en una ocasión (27 de julio de 2016) una vez que ya el SEPE había decidido unilateralmente la prórroga y teniendo en cuenta que la normativa que lo regula establece que como mínimo la Comisión ha de reunirse dos veces al año.

7 MAQUEDA, A. y GÓMEZ, M. 2017. "La justicia frena el plan de Empleo que usa agencias privadas para recolocar parados". El País. [25-01-2017] (http://economia.elpais.com/economia/2017/01/23/actualidad/1485204362_824201.html?id_externo_ rSOC=TW_CC).

${ }^{\circ}$ MAQUEDA, A. 2016. "La colocación público-privada de parados, más eficiente que las oficinas de empleo". El País. [06-092016] (http://economia.elpais.com/economia/2016/08/27/actualidad/1472331035_747571.html). 


\section{EL CASO DE LA COMUNIDAD VALENCIANA: UN CAMINO DE IDA Y VUELTA}

La Comunidad Valenciana, completó el traspaso de competencias en materia de políticas activas de empleo en 2000 con la aprobación de la ley de creación del Servef y fue una de las comunidades que en 2013 suscribió el convenio con el SEPE para adherirse al Acuerdo Marco. Con un gobierno del PP todavía en el poder, en enero de 2014 se aprobó el Plan Estratégico SERVEF en el que la CPP se configuraba como pilar clave de la estrategia del Servef (SERVEF 2014). En octubre de 2014, se confirmaba el inicio del procedimiento para la licitación del primer contrato en el ámbito autonómico, entre el Servef y las agencias colaboradoras, derivado del Acuerdo Marco ${ }^{9}$. Todos los partidos en la oposición mostraron su disconformidad con lo que consideraban una privatización del servicio de intermediación laboral ${ }^{10}$.

En mayo de 2015 se celebraron las elecciones autonómicas de las que salió un nuevo gobierno de coalición entre el PSOE y Compromís (con el apoyo de Podemos en el llamado Pacto del Botánico), sin que hubiera finalizado la licitación de ese primer contrato del Servef con las agencias colaboradoras privadas. Conviene recordar en este punto que estos partidos que antes estaban en la oposición habían sido tradicionalmente muy duros con este tipo de iniciativas privatizadoras de la gestión también en otros sectores de política pública como la sanidad (Acerete, Stafford, y Stapleton 2011; Del-Pino y Catalá-Pérez 2016). Con la formación del nuevo gobierno, se empezó a vislumbrar el cambio de estrategia en numerosos áreas de política pública y también en materia de intermediación. El área de empleo del nuevo ejecutivo autonómico sugería que iba a comenzar una nueva etapa, en la que la administración local en general, y los agentes de ocupación y desarrollo local en particular, estaban llamados a incrementar el protagonismo en la implementación de las políticas activas ${ }^{11}$.

A finales de 2015, directivos del Servef confirmaban que el procedimiento de licitación del primer contrato autonómico derivado del Acuerdo Marco seguía en marcha pero que se había retrasado por el cambio de gobierno autonómico y por la detección de irregularidades en los procesos de inspección, pero que antes de finalizar el año habría adjudicación definitiva ${ }^{12}$. Finalmente, en el mes de diciembre se confirmaba la paralización del procedimiento de licitación del citado contrato y la desvinculación de la Comunidad Valenciana del Acuerdo. Cabe señalar, según se ha recabado en las entrevistas, que la licitación del que hubiera sido ese primer contrato de colaboración en el ámbito valenciano, estaba muy cerca de su resolución. Se había abierto el sobre 2 con lo que se habían llegado a preseleccionar varias agencias de colocación, de entre las que saldrían las adjudicatarias finales. El contrato se dividía en nueve lotes y presentaba características innovadoras como el hecho de tener en cuenta a las agencias de colocación telemáticas y la atención que se prestaba al control y la inspección de la actividad de las agencias.

El nuevo gobierno explicaba que se pretendía aprobar una orden propia en materia de colaboración entre el Servef y las agencias de colocación, siguiendo el ejemplo de Cataluña. Además se confirmaba que solo si el Servef no tenía la suficiente capacidad, se daría acceso a entidades del ámbito privado, ya que se consideraba que el sistema creado por el PP era «una fórmula de negocio para externalizar lo rentable». La comisión de valoración creada para estudiar la conveniencia o no de continuar con la estrategia de CPP marcada por el PP, consideraba que las agencias privadas duplicaban servicios del Servef o de las propias

9 JIMENO, G. 2014. "El Servef revoluciona su gestión para ofrecer servicios de valor a ciudadanos y empresas". Economía3. [28-12-2016] (http://www.economia3.com/2014/10/20/32030-el-servef-revoluciona-su-gestion-para-ofrecer-servicios-de-valor-a-ciudadanos-y-empresas/)

10 LIGORIO, C. 2013. "El Servef busca «colaboradores» privados". Valencia News. [28-12-2016] (http://valencianews.es/economia/empresa/el-servef-busca-colaboradores-privados/).

11 Según se desprende de las declaraciones de los dirigentes del Servef publicadas en prensa:

- AGENCIA EFE. 2015b. "La Conselleria de Economía mantendrá a los Agentes de Desarrollo Local". La Vanguardia. [29-122016] (http://www.lavanguardia.com/local/valencia/20150913/54436482684/la-conselleria-de-economia-mantendra-a-los-agentes-de-desarrollo-local.html).

- EL PERIÒDIC. 2015. "Rubén Alfaro se reúne con el Servef para impulsar políticas activas de empleo". El Periòdic. [04-01-2017] (http://www.elperiodic.com/elda/noticias/405383 ruben-alfaro-reune-servef-para-impulsar-politicas-activas-empleo.html).

- JOBSHUNTERS. 2015a. "Servef contará con administraciones locales para políticas activas de empleo". JobsHunters-Revista Agencias de Colocación y Empleo. [29-12-2016] (http://www.jobshunters.es/servef-contara-con-administraciones-locales-parapoliticas-activas-de-empleo/).

12 JOBSHUNTERS. 2015b. "ANAC se reúne en Valencia con SERVEF y el Ayuntamiento: «La colaboración público-privada es una apuesta que se pondrá en marcha»". JobsHunters-Revista Agencias de Colocación y Empleo. [07-01-2017] (http://www.jobshunters.es/anac-se-reune-en-valencia-con-servef-y-el-ayuntamiento-la-colaboracion-publico-privada-es-una-apuesta-que-se-pondra-enmarcha). 
agencias de desarrollo local ${ }^{13}$. Además, según un representante del Servef, otro de los argumentos aducidos fue que los resultados obtenidos por las agencias de colocación no mejoraban los obtenidos por el Servef en materia de intermediación.

En este sentido, los resultados a los que se hizo referencia en las entrevistas eran los presentados por el Ministerio de Empleo en un informe ya mencionado y dado a conocer en agosto de 2016, relativos al primer contrato de ámbito estatal licitado por el SEPE para la colocación de los parados del Plan Prepara ${ }^{14}$.Según estos datos, un total de 189.878 personas desempleadas fueron enviadas a las agencias adjudicatarias durante el primer año del contrato, de las cuales fueron atendidas 81.638 (un 43\%); y de estas, se colocó a un total de 8.051, alcanzando una tasa de inserción del 4,2\% de las enviadas y del 9,9\% de las atendidas (Jansen 2016:59) ${ }^{15}$. En febrero de 2017, la ministra de Empleo, Báñez, ofrecía datos más actualizados según los cuales a través de la CPP la inserción media de las personas atendidas llegaba al 10,7\%, situándose en el 14\% en el caso de agencias contratadas por el SEPE (el $18 \%$ en el caso de tener en cuenta únicamente a los beneficiarios del Plan Prepara) y en el $8 \%$ en el caso de aquellas agencias privadas de colocación contratadas por las CCAA ${ }^{16}$.

Negueruela (2016) ha criticado que estos datos del informe de agosto de 2016 puedan calificarse de positivos. El hecho de que las agencias de colocación atendieran únicamente al $43 \%$ de las personas enviadas es, para el autor, motivo para inhabilitar a las agencias como colaboradoras. En segundo lugar, Negueruela considera que las agencias, por su forma de funcionar, no realizan una captación de ofertas ni una selección de demandantes reales, que sería la verdadera intermediación, sino que según sus propias palabras «dan palos de ciego y, si suena la flauta, se anotan un tanto y cobran por él». Por ello considera que las cifras de las agencias se deben comparar con los datos del total de bajas por colocación del SNE y no únicamente con las colocaciones con oferta previa. Según este criterio, tomando datos del SEPE, en 2015 hubo 5.721 .430 demandantes inscritos que fueron baja por colocación, una media de 476.786 bajas al mes que relacionados con una media de 5.591 .745 demandantes mensuales, dan un porcentaje medio de colocaciones del $8,53 \%$ sobre el número de personas inscritas (que sería el dato equivalente al de personas enviadas a las agencias de colocación), es decir, 4,4 puntos por encima de las agencias de colocación según los datos anteriores.

Siguiendo este criterio, a partir de los datos del SEPE para la Comunidad Valenciana, y tomando en consideración el período temporal correspondiente al primer año del contrato estatal para colocar a los parados del Plan Prepara (es decir, de agosto de 2015 a julio de 2016), se registró una media mensual de 595.684 demandantes de empleo, para un total de 530.855 bajas por colocación, a razón de 44.238 mensuales, lo que ofrece un porcentaje medio de colocaciones del 7,43\% por parte del Servef, ligeramente por debajo del dato equivalente estatal, pero un $3,2 \%$ por encima de las agencias privadas.

Por otra parte, teniendo en cuenta que el período revisado para hacer los cálculos anteriores cubre los meses que van de agosto a diciembre de 2015, cuando aún el gobierno valenciano no había paralizado definitivamente el proyecto de CPP, tal vez resulte interesante eliminar estos meses y hacer los cálculos del porcentaje de colocaciones conseguidas por el Servef durante todo el 2016, primer año completo de gestión del nuevo gobierno, con la nueva estrategia del Servef ya definida. De esta forma, los porcentajes que se obtienen apenas varían: el Servef alcanza un 7,65\% de bajas de demandantes por colocación (una media de 43.846 bajas mensuales sobre un número medio mensual de 573.060 demandantes de empleo).

En cualquier caso, lo que podría ser más interesante es comparar los datos del Servef con los obtenidos por las agencias de colocación colaboradoras que operaban en la Comunidad Valenciana en virtud de los dos contratos estatales firmados por el SEPE. En este sentido, fruto de las entrevistas con representantes del sector privado se tuvo acceso a un informe de resultados de «T de Talento», que es la agencia de colocación adjudicataria del lote núm. 2 del primer contrato estatal del SEPE (el de los desempleados del Plan Prepara). Este contrato se adjudicó en lotes dentro de cada uno de los cuales se encontraban unas determinadas CCAA y en concreto, en el mencionado lote núm. 2, se encontraba la Comunidad Valenciana, Murcia y Baleares.

Con la cautela requerida al analizar datos procedentes de la propia empresa, existen diferencias reseñables entre los porcentajes de colocación que «T de Talento» aseguraba haber conseguido en la Co-

13 ÁLVAREZ, F. 2015. "Mira: «Queremos elevar los salarios y dar estabilidad a los interinos del Servef»”. El Mundo. [05-01-2017] (http://www.elmundo.es/comunidad-valenciana/2015/12/27/567f0459ca4741a6318b458a.html).

14 Dado que la experiencia de colaboración del gobierno valenciano con el sector privado mediante un contrato autonómico no llegó a materializarse, no había resultados de experiencias previas en la Comunidad Valenciana.

15 Estos datos fueron inicialmente publicados por Maqueda, art. cit., 2016.

16 EUROPA PRESS. 2017. "Báñez dice que la CPP hace que la tasa de inserción laboral sea del 10,7\%". Europa Press. [16-022017] (http://www.europapress.es/economia/laboral-00346/noticia-banez-dice-colaboracion-publico-privada-hace-tasa-insercion-laboralsea-107-20170207183414.html). 
munidad Valenciana durante el primer año de contrato y los reflejados para todo el país en el informe de resultados presentado por el Ministerio de Empleo. Estaríamos hablando de que el SEPE remitió a «T de Talento» en la Comunidad Valenciana a un total de 4.567 personas, de las que fueron atendidas 3.020 (un $66 \%$ de las remitidas, superando en un $23 \%$ el porcentaje de personas atendidas a nivel estatal). Se consiguió colocar a un total de 898 personas, lo que supone un $19,66 \%$ de las personas remitidas y un $29,74 \%$ de las atendidas por la agencia. Nuevamente, hay que ser cautelosos con estos datos tanto por su procedencia como porque habría que conocer otra información de contexto. En cualquier caso, los resultados mejorarían sensiblemente los obtenidos por la CPP a nivel estatal (recordemos, el $4,2 \%$ de las personas enviadas y el $9,9 \%$ de las atendidas).

Los resultados de la CPP en cuanto a cobertura son muy relevantes para saber si la decisión de recurrir o no a ella está justificada o no, pero igualmente lo es la medida en que su aplicación puede afectar a la calidad del servicio prestado. Parece que el sistema público no cuenta con los recursos humanos necesarios ni los incentivos adecuados para ofrecer servicios individualizados de calidad a todos los parados y por ello, el uso de las agencias de colocación debería permitir un aumento de los recursos disponibles y un mayor grado de especialización que actualmente no existe (Bentolilla y Jansen 2013). Pero también en este sentido, Negueruela ha denunciado $(2013,2015,2016)$ la baja calidad de los servicios de intermediación prestados por las agencias de colocación colaboradoras en el caso de Galicia: escasa o nula atención personalizada a los desempleados a los que llaman generalmente desde teléfonos privados en horarios muchas veces intempestivos para convocarlos a citas en salones de hotel o pisos particulares, en donde se les facilitan listados generales de ofertas sin ningún tipo de filtro según circunstancias personales o simplemente, las claves para acceder a un portal de internet sin más información; y todo esto llevado a cabo por entidades que no disponen de oficinas propias o que ni siquiera operan habitualmente en la comunidad autónoma.

También en la Comunidad Valenciana se han producido algunos episodios que han generado desconcierto entre los desempleados. Al empezar a operar en agosto de 2015, «T de Talento» inició un sondeo de posibles candidatos a través de SMS sin que los parados hubieran recibido ningún tipo de comunicación oficial previa. Esto generó desconfianza y múltiples consultas al Servef que confirmó que se trataba de una acción que partía del SEPE de la que no se tenía conocimiento por su parte (ADLYPSE 2015).

Algunas de las entrevistas realizadas ${ }^{17}$ han confirmado la falta de coordinación existente entre los diferentes actores que participan en la prestación del servicio de intermediación y colocación laboral. Las agencias de colocación autorizadas, privadas o públicas (generalmente Agencias de Desarrollo Local ADL) reportan la información relativa a su funcionamiento directamente al SEPE, ya estén autorizadas por el propio SEPE (si operan en diferentes CCAA) o por el Servef (si operan únicamente en la Comunidad Valenciana), de forma que, en la práctica, el Servef no tiene comunicación con las agencias de colocación que operan en su territorio. Puede darse el caso, por ejemplo, de que una persona inscrita en el Servef, sea en realidad atendida por la ADL de su localidad, que a su vez le recomiende inscribirse en determinada ETT y que en este proceso reciba una comunicación del SEPE (por ejemplo, a través de «T de Talento») para acudir a una entrevista, sin que ninguna de estas cuatro entidades tenga conocimiento de las acciones llevadas a cabo por las otras tres sobre dicha persona.

Está situación puede producir desconcierto en los desempleados e incluso perjudicarles si el servicio ofrecido y el trato recibido en cada caso varía. En las entrevistas surgió algo interesante en este sentido y es que dada la heterogeneidad del tipo de organizaciones a las que se ha autorizado como agencias de colocación (ETT, empresas de formación, organizaciones sindicales y empresariales, despachos de abogados, fundaciones, entidades de carácter religioso, asociaciones y fundaciones, etc...), es probable que en algunos casos, el personal no sea experto en intermediación laboral y no puedan ofrecer un servicio de calidad como el que se supone al personal experto de los servicios públicos de empleo.

\section{DISCUSIÓN Y CONCLUSIONES}

Aunque desde hace tres décadas en los países occidentales, incluida España, el Estado comenzó a contar cada vez con más frecuencia con el sector privado para la provisión de los servicios del bienestar, la introducción de estas fórmulas no ha sido siempre sencilla. En el sector de las políticas activas de empleo,

\footnotetext{
17 Tanto desde el sector público como del privado.
} 
España empezó a utilizar fórmulas de este tipo recientemente. La reforma de la gobernanza de la intermediación laboral en nuestro país no ha estado tampoco exenta de dificultades. En este artículo se ha analizado este caso específicamente en la Comunidad Valenciana utilizando las claves que ofrece la literatura científica respecto a los factores que explican el alcance de los cambios en la gobernanza de los sistemas de protección social y, en concreto, de las políticas activas de empleo para entender estas dificultades. Este análisis aporta nueva evidencia empírica a esta literatura y sirve para entender los problemas de reforma de estas iniciativas en el caso de España.

En el caso analizado, asistimos a un doble giro en la gobernanza del servicio de intermediación laboral en la Comunidad Valenciana. Gobiernos nacionales de diferente ideología llevaron a cabo reformas en el sector de la intermediación laboral siguiendo las recomendaciones de numerosos expertos así como de organizaciones internacionales con el objetivo de incrementar la participación del sector privado en la provisión del servicio de intermediación laboral. Estas reformas liberalizaban en cierta medida un servicio incluido en el ámbito de las políticas activas de empleo, de competencia autonómica. Las reformas dieron la opción a los gobiernos autonómicos de adherirse a un Acuerdo Marco con el que se pretendía sentar las bases de la colaboración entre los SPE y las agencias de colocación privadas.

En una primera fase, un gobierno autonómico valenciano del mismo color que el Gobierno Central inició a finales de 2014, el procedimiento de contratación para implementar la colaboración entre los servicios de empleo regionales y las agencias de colocación previamente seleccionadas. Además, el servicio de empleo nacional adjudicó la colocación de los parados con especiales dificultades en la Comunidad Valenciana hasta octubre de 2017.

En una segunda fase, tras las elecciones autonómicas de mayo del 2015, el nuevo gobierno compuesto por una coalición de partidos de izquierda modificó la estrategia regional en materia de intermediación y colocación, desvinculándose del ministerio, algo parecido a lo que ha ocurrido en otras CCAA que también cambiaron el signo político en sus gobiernos después de las mencionadas elecciones, pasando a gobernar el PSOE u otras coaliciones de izquierdas. Finalmente, al menos en el caso del gobierno autonómico valenciano no se ha contratado con las Agencias de Colocación Colaboradoras y en el momento de acabar este texto, no parece que vaya a hacerlo.

¿Cuál ha sido finalmente el alcance de los cambios? ¿Qué factores pueden explicarlos? Por lo que se refiere al alcance de los cambios, la reforma inicial diseñada por el Gobierno central fue mucho más ambiciosa que la finalmente implementada. Varios factores pueden explicar esta situación. Aunque no existía un consenso absoluto sobre las soluciones a los problemas de la intermediación laboral en España, los malos resultados en esta materia por parte de los servicios públicos de empleo, como también, el grave problema del desempleo en España exigían una respuesta del gobierno. En el marco del paradigma del Estado orientado a la Inversión Social, las políticas activas de empleo y la intermediación laboral se han reformado y fortalecido en la mayoría de los gobiernos europeos. Además, en los últimos años, en el marco de la NGP, es creciente la apuesta por la CPP para prestar estos servicios de intermediación. En España, estas ideas parecen también haber calado en gobiernos de distinto color político ya que han ido progresivamente apostando por las políticas activas y el aumento del papel del sector privado en la intermediación laboral, aunque fue un gobierno conservador el que dio el paso definitivo y más claro hacia el cambio en la gobernanza. El contexto de profunda crisis impulsó también esta decisión, ya que en ese contexto los gobiernos encuentran más difícil permanecer inactivos y esta era una propuesta largamente aconsejada por numerosos expertos y organizaciones internacionales.

Por otro lado, como prevé la literatura académica, las reformas en las que intervienen varios niveles de gobierno pueden ser complejas y más en el caso de que estos estén ocupados por partidos con diferente color político y con diferentes ideas sobre la gestión de las políticas sociales. Si los gobiernos subnacionales no encuentran suficiente atractivo en la oferta del gobierno que promueve las reformas, la implementación puede complicarse. Igualmente, como no es infrecuente observar en otros procesos de implementación de este tipo de iniciativas de política pública, la participación de otros actores no públicos, numerosos y diversos, en este caso empresas de diferentes características e intereses, puede hacer aún más compleja la puesta en marcha de estas iniciativas, especialmente cuando la experiencia de la administración era tan escasa como en este caso. No es infrecuente que las administraciones pasen por un proceso de ensayo y error al introducir esas fórmulas de provisión de los servicios y muchas veces estos problemas lastran para siempre estas experiencias (véase por ejemplo, el caso de Alzira) ya que los fallos son utilizados por los críticos. En este sentido, la administración debería aprovechar lo aprendido en este tipo de procesos y evitar que se repitieran errores similares una y otra vez. Para ello debería mejorarse el marco regulatorio e institucional de la CPP que presenta algunos fallos en nuestros país. 
Además, aunque de acuerdo con la literatura académica, independientemente de su color, los gobiernos utilizan crecientemente mecanismos de provisión privada de los servicios públicos, en el caso analizado hemos encontrado algunos matices. Mientras en la puesta en marcha de la iniciativa analizada estuvieron involucrados gobiernos de diferentes ideologías, lo cierto es que en buena parte del limitado alcance final de esta medida parece haberse debido a discrepancias ideológicas sobre qué papel deben desempeñar la administración y del sector privado en la provisión de servicios públicos. En el caso analizado la oposición a la privatización de la gestión de los servicios públicos se había además convertido en una seña de identidad de la izquierda durante el largo periodo de gobierno conservador del PP en esta comunidad autónoma.

Por un lado, en el caso analizado, quienes respaldaban la participación del sector privado apoyan su propuesta en los buenos resultados que estas experiencias han tenido en otros países, así como en los datos positivos que arrojan los resultados provisionales existentes en España. Quienes, por el contrario, se oponen a este tipo de instrumentos utilizan el argumento de que si los resultados de los servicios de empleo son peores en ocasiones, ello se debe a una progresiva merma de sus capacidades técnicas y humanas derivadas de la falta de voluntad política y, en los últimos años, de las políticas de austeridad; y denuncian su deficiente regulación y que los mecanismos previstos en las normas no son suficientes para garantizar con solidez que la cobertura, la calidad del servicio o su eficacia sean los que a su juicio cabría esperar de un sistema avanzado de protección social. $\mathrm{Si}$, como con frecuencia ocurre, no existen datos objetivos fruto de evaluaciones rigurosas para poder rebatir unos u otros argumentos, están posturas seguirán sin reconciliarse.

\section{BIBLIOGRAFÍA}

ACERETE, B., STAFFORD, A., y STAPLETON, P. 2011. "Spanish healthcare public private partnerships: The «Alzira model»". Critical Perspectives on Accounting, 22 (6): 533-549. DOI: https://doi.org/10.1016/j.cpa.2011.06.004.

ADLYPSE. 2015. "EI SEPE y sus acuerdos de colaboración con las Agencias de Colocación". adlypse.org. [28-122016] (http://adlypse.org/?p=2382).

ALUJAS, J. A. 2008. "La eficacia del Servicio Público de Empleo en España. Análisis de la intermediación laboral a nivel autonómico". Información Comercial Española, ICE: Revista de economía (841): 167-80.

ASCOLI, U. y RANCI, C., eds. 2002. Dilemmas of the Welfare Mix: The New Structure of Welfare in an Era of Privatization. New York: Springer US.

BENTOLILLA, S. y JANSEN, M. 2013. "Cooperación público-privada en la reinserción de los parados". Nada es gratis. [06-09-2016] (http://nadaesgratis.es/bentolila/cooperacion-publico-privada-en-la-reinsercion-de-los-parados).

VAN BERKEL, R., DE-GRAAF, W., y SIROVÁTKA, T. 2011. "The Governance of Active Welfare States". págs. 1-21 en The Governance of Active Welfare States in Europe, editado por W. DE-GRAAF, R. VAN BERKEL, Y T. SIROVÁTKA. London: Palgrave Macmillan UK. DOI: https://doi.org/10.1057/9780230306714_1.

BONOLI, G. 2011. "Active Labour Market Polity in a changing Economic Context". págs. 318-332 en Regulating the Risk of Unemployment National Adaptations to Post-Industrial Labour Markets in Europe, editado por J. CLASEN y D. CLEGG. Oxford: Oxford University Press.

BORTOLOTTI, B., FANTINI, M., y SINISCALCO, D. 2004. "Privatisation around the world: evidence from panel data". Journal of Public Economics 88 (1-2): 305-332. DOI: https://doi.org/10.1016/s0047-2727(02)00161-5.

BREIDAHL, K. N. y LARSEN, F. 2015. "The Developing Trajectory of the Marketization of Public Employment Services in Denmark-A New Way Forward or the End of Marketization?" European Policy Analysis 1 (1): 92-107. DOI: https://doi.org/10.18278/epa.1.1.7.

BRIDGEN, P. y MEYER, T. 2008. "Politically Dominant but Socially Flawed: Projected Pension Levels for Citizens at Risk in Six European Multi-Pillar Pension Systems". págs. 111-131 en Welfare State Transformations. Comparative Perspectives, editado por M. Seeleib-Kaiser. London: Palgrave Macmillan UK. DOI: https://doi. org/10.1057/9780230227392_7.

CALVO-GALLEGO, F. J. 2011. "Transiciones laborales y nuevo modelo productivo: Algunas notas sobre la incidencia de la reforma laboral de 2010". págs. 39-138 en Relaciones laborales y nuevo modelo productivo, editado por J. CASTIÑEIRA FERNÁNDEZ. Sevilla: Consejo Andaluz de Relaciones Laborales.

CALVO-GALLEGO, F. J. y RODRÍGUEZ-PIÑERO, M. 2010. "Nuevas normas en materia de intermediación y empresas de trabajo temporal". Temas laborales: Revista andaluza de trabajo y bienestar social (107): 303-335.

CALVO, R. y LERMA, J. I. 2009. "Políticas activas de empleo en tiempo de crisis: una visión crítica desde lo local". Lan harremanak: Revista de relaciones laborales (20): 227-250.

CCOO. 2016. "El Gobierno prorroga los contratos de las agencias privadas de colocación sin realizar evaluación". ccoo.es. [07-01-2017] (http://www.ccoo.es/noticia:228403--El_Gobierno_prorroga_los_contratos_de_las_ agencias_privadas_de_colocacion_sin_realizar_evaluacion). 
CLASEN, J. y CLEGG, D. 2011. Regulating the risk of unemployment: national adaptations to post-industrial labour markets in Europe. Oxford: Oxford University Press.

COLINO, C. y DEL PINO, E. 2015. "National and European patterns of public administration and governance". págs. 611-639 en Routledge Handbook of European Politics, editado por J. M. Magone. London: Routledge.

CONSEJO ECONÓMICO Y SOCIAL. 2014. Informe sobre la Situación Sociolaboral de las Personas de 45 a 64 años de edad. Madrid: Publicaciones CES.

DE-LA-PORTE, C. y HEINS, E. 2014. "A New Era of European Integration? Governance of Labour Market and Social Policy since the Sovereign Debt Crisis". Comparative European Politics 13 (1): 8-28.

DE-LA-RICA, S. 2015. "Políticas Activas de Empleo: Una Panorámica". Policy Papers 1-32.

DE-VICENTE PACHÉS, F. 2012. "Intermediación laboral y agencias de colocación". págs. 377-405 en Las reformas laborales del 2010: Jornadas Catalanas de Derecho Social, editado por J. MORENO I GENÉ, A. SOLÉ PUIG, y C. L. ALFONSO MELLADO. Lleida: Huygens.

DE-VICENTE PACHÉS, F. 2013. "Las agencias de colocación mediante la forma jurídica de empresa de economía social". Revista de treball, economia i societat (68): 1-32.

DEL-PINO, E. y CATALÁ-PÉREZ, D. 2016. "El welfare-mix español durante la crisis y la privatización del riesgo social: Ios casos de la sanidad, los servicios sociales y la atención a la dependencia". Revista del CLAD Reforma y Democracia (66): 163-194.

DEL-PINO, E. y RAMOS, J. A. 2016. "Políticas de protección por desempleo en perspectiva comparada: hacia la remercantilización y la activación”. págs. 293-316 en Los Estados de bienestar en la encrucijada: políticas sociales en perspectiva comparada, editado por E. DEL PINO y M. J. RUBIO LARA. Madrid: Tecnos.

EUROFOUND. 2015. Delivering public services: A greater role for the private sector? An exploratory study in four countries. editado por Eurofund. Luxemburg: Publications Office of the European Union.

FERNÁNDEZ, C. J. y MARTíN, M. P. 2014. "Los discursos sobre la modernización de los Servicios Públicos de Empleo: ¿hacia una nueva forma de gobernanza?" Política y Sociedad 51 (1): 177-200.

FUNDACIÓN ÉLOGOS, ed. 2012. Reflexiones sobre el marco de colaboración público-privada en España en el ámbito del empleo. Madrid: Fundación Élogos.

GINGRICH, J. R. 2011. Making markets in the welfare state: the politics of varying market reforms. Cambridge: Cambridge University Press. DOI: https://doi.org/10.1017/CBO9780511791529.

GRAZIANO, P. R. 2012. "Converging worlds of activation?" editado por R. van Berkel. International Journal of Sociology and Social Policy 32 (5/6): 312-326.

GRAZIANO, P. R. y RAUÉ, A. 2011. "The Governance of Activation Policies in Italy: from Centralized and Hierarchical to a Multi-Level Open System Model?" págs. 110-131 en The Governance of Active Welfare States in Europe, editado por W. De Graaf, R. VAN BERKEL, y T. SIROVÁTKA. London: Palgrave Macmillan UK. DOI: https://doi. org/10.1057/9780230306714_6.

GREER, S. L. 2010. "How Does Decentralisation Affect the Welfare State? Territorial Politics and the Welfare State in the UK and US". Journal of Social Policy 39 (2): 181-201. DOI: https://doi.org/10.1017/s0047279409990407.

GREVE, B. 2015. Welfare and the Welfare State: Present and Future. Oxon: Routledge. DOI: https://doi. org/10.4324/9781315761022.

GREVE, C. y HODGE, G. 2013. Rethinking public-private partnerships: strategies for turbulent times. Abingdon: Routledge. DOI: https://doi.org/10.4324/9780203108130.

HEIDENREICH, M. y AURICH-BEERHEIDE, P. 2014. "European worlds of inclusive activation: The organisational challenges of coordinated service provision". International Journal of Social Welfare 23 (S1): S6-22. DOI: https:// doi.org/10.1111/ijsw.12098.

HERNÁNDEZ-MORENO, J. J. y RAMOS, J. A. 2017. "La centralización de la gobernanza territorial en las políticas activas del mercado de trabajo en España durante la Gran Recesión (2011-2016)". Gestión y Análisis de Políticas Públicas (18). DOI: https://doi.org/10.24965/gapp.v0i18.10433.

HOOGEMBOOM, M. 2011. "The Netherlands: two tiers for all". págs. 75-99 en Regulating the Risk of Unemployment National Adaptations to Post-Industrial Labour Markets in Europe, editado por J. CLASEN y D. CLEGG. Oxford: Oxford University Press. DOI: https://doi.org/10.1093/acprof:oso/9780199592296.003.0005.

JANSEN, M. 2016. El reto de la inserción de los desempleados de larga duración. Madrid.

JANSEN, M. 2017. "Razones equivocadas para frenar a las agencias de colocación - Nada es Gratis". Nada es Gratis. [30-01-2017] (http://nadaesgratis.es/marcel-jansen/razones-equivocadas-para-frenar-a-las-agencias-decolocacion).

KAZEPOV, Y. 2010. Rescaling Social Policies: Towards Multilevel Governance in Europe. Surrey: Ashgate Publishing, Ltd.

KENWORTHY, L. 2010. "Labour Market Activation”. págs. 435-448 en The Oxford Handbook of the Welfare State, editado por F. G. CASTLES, S. LEIBFRIED, J. LEWIS, H. OBINGER, y C. PIERSON. Oxford: Oxford University Press. DOI: $h$ ttps://doi.org/10.1093/oxfordhb/9780199579396.003.0030.

KLENK, T. 2018. "Welfare Administration and Its Reform". págs. 727-744 en The Palgrave Handbook of Public Administration and Management in Europe. London: Palgrave Macmillan UK. DOI: https://doi.org/10.1057/978-1137-55269-3_38. 
GAPP. Nueva Época - N.1, mayo 2018 - ISSN: 1989-8991 - DOI: 10.24965/gapp.v0i19.10434 - [Págs. 107-122]

Reformas de ida y vuelta en la gobernanza horizontal del bienestar: el caso de la colaboración público-privada en la..

Daniel Catalá Pérez / Eloísa del Pino

KLENK, T. y PAVOLINI, E. 2015. Restructuring Welfare Governance Marketisation, Managerialism and Welfare State Professionalism. Cheltenham: Edward Elgar Publishing Ltd.

KRUMM, T. 2016. The Politics of Public-Private Partnerships in Western Europe. Comparative Perspectives. Cheltenham: Edward Elgar Publishing Ltd. DOI: https://doi.org/10.4337/9781782549260.

LÓPEZ-SANTANA, M. 2015. The new governance of welfare states in the United States and Europe: between decentralization and centralization in the activation era. Albany, NY: Suny Press.

LÓPEZ-SANTANA, M. 2016. "Regional and Domestic Responses to the Unemployment Problem in Europe: Reconfiguring the Architectures of Welfare States". págs. 117-138 en Regional Organizations and Social Policy in Europe and Latin America, editado por A. C. BIANCULLI y A. R. HOFFMANN. London: Palgrave Macmillan UK. DOI: $h$ ttps://doi.org/10.1057/9781137490353_6.

MARTIN, J. P. 2015. "Activation and active labour market policies in OECD countries: stylised facts and evidence on their effectiveness". IZA Journal of Labor Policy 4 (1): 4. DOI: https://doi.org/10.1186/s40173-015-0032-y.

MATHIAS, J. 2017. "Reforming the Swedish employment-related social security system: activation, administrative modernization and strengthening local autonomy". Regional \& Federal Studies 27 (1): 23-39. DOI: https://doi.org/1 $0.1080 / 13597566.2016 .1255605$.

MERCADER-UGUINA, J. R. 2014. Se busca...: el mercado de trabajo en España. Barcelona: Debate.

MINISTERIO DE EMPLEO Y SEGURIDAD SOCIAL. 2015. "EI Gobierno aprueba un contrato del SEPE con Agencias de Colocación basado en el Acuerdo Marco de Intermediación Laboral". Ministerio de Empleo y Seguridad Social. Gabinete de Comunicación. [28-12-2016] (http://prensa.empleo.gob.es/WebPrensa/noticias/ministro/detalle/2536).

MOLINA-NAVARRETE, C. 2012. "Intermediación y políticas activas de empleo: Una urgente reforma «que brilla por su ausencia»". Temas laborales: Revista andaluza de trabajo y bienestar social (115): 135-164.

MULLOR, M. 2011. "La reforma de los servicios públicos de empleo". Cuadernos de pensamiento político FAES (29): $167-178$.

NEGUERUELA, E. 2013. "Colaboración público-privada en empleo: parados en venta y sancionadores a comisión". Nueva Tribuna. [27-12-2016] (http://www.nuevatribuna.es/opinion/enrique-negueruela/colaboracion-publicoprivada-en-empleo-parados-en-venta-y-sancionadores-a-comision/20130623125557093923.html).

NEGUERUELA, E. 2015. "Agencias privadas de colocación: sancionadores a comisión”. Nueva Tribuna. Recuperado [07-01-2017] (http://www.nuevatribuna.es/articulo/economia-social/agencias-privadas-colocacion-sancionadorescomision/20151202081710122962.html).

NEGUERUELA, E. 2016. "El balance hecho por el Gobierno sobre las agencias privadas de colocación es un timo". Nueva Tribuna. [07-01-2017] (http://www.nuevatribuna.es/articulo/economia-social/timo-agencias-privadascolocacion/20161013123151132632.htm/).

NIETO-ROJAS, P. 2012. "La dimensión local de las políticas activas de empleo. Una revisión competencial tras la aprobación de la estrategia española de empleo 2012-2014". Temas laborales: Revista andaluza de trabajo y bienestar social (114): 119-146.

OBINGER, H. y STARKE, P. 2014. Welfare state transformation: Convergence and the rise of the supply side model. Bremen: University of Bremen.

OOSTERLYNCK, S. et al. 2013. Exploring the multi-level governance of welfare provision and social innovation: welfare mix, welfare models and rescaling. Amberes: Herman Deleeck Centre for Social Policy, University of Antwerp.

PARREÑO, V. 2015. "El papel de los servicios en la construcción del territorio: redes y actores". págs. 257-270 en El papel de los servicios en la construcción del territorio: redes y actores, editado por A. ESPINOSA SEGUÍ y F. J. ANTÓN BURGOS. Alicante: Asociación de Geógrafos Españoles.

PIERSON, P. 2001. The new politics of the welfare state. editado por P. PIERSON. Oxford: Oxford University Press. DOI: https://doi.org/10.1093/0198297564.001.0001.

PLANTINGA, M., de RIDDER, K., y CORRA, A. 2011. "Choosing Whether to Buy or Make: The Contracting Out of Employment Reintegration Services by Dutch Municipalities”. Social Policy \& Administration 45 (3): 245-263. DOI: https://doi.org/10.1111/j.1467-9515.2011.00767.x.

POLLITT, C. y BOUCKAERT, G. 2011. Public management reform: a comparative analysis: new public management, governance, and the neo-Weberian state. Oxford: Oxford University Press.

PWC-ASEMPLEO. 2014. "La Colaboración Público-Privada en el Mercado de Trabajo: Experiencias Comparadas y Posibilidades para España".

RANSTAD RESEARCH, ed. 2015. Informe sobre modelos internacionales de éxito en colaboración público-privada. Madrid: Ranstad Research.

RODRÍGUEZ-PIÑERO, M. 2014. "Aproximación a la colaboración público-privada en el mercado de trabajo español”. Temas laborales: Revista andaluza de trabajo y bienestar social (125): 53-87.

ROTHGANG, H., CACACE, M., FRISINA, L., y SCHMID, A. 2008. "The Changing Public-Private Mix in OECD Health-care Systems". págs. 132-146 en Welfare State Transformations. London: Palgrave Macmillan UK. DOI: https://doi.org/10.1057/9780230227392_8.

SÁNCHEZ-RODAS, C. 2014. "La orientación e intermediación directa en el empleo". Temas laborales: Revista andaluza de trabajo y bienestar social (125): 89-113. 
GAPP. Nueva Época - N.19, mayo 2018 - ISSN: 1989-8991 - DOI: 10.24965/gapp.v0i19.10434 - [Págs. 107-122]

Reformas de ida y vuelta en la gobernanza horizontal del bienestar: el caso de la colaboración público-privada en la...

Daniel Catalá Pérez / Eloísa del Pino

SEELEIB-KAISER, M., ed. 2008. Welfare State Transformations. Comparative perspectives. London: Palgrave Macmillan UK.

SERVEF. 2014. Plan Estratégico SERVEF 2014-2020. Valencia: Generalitat Valenciana.

VAN-BERKEL, R. y BORGHI, V. 2008. "Review Article: The Governance of Activation". Social Policy and Society 7 (3): 393-402. DOI: https://doi.org/10.1017/s1474746408004351.

WEISHAUPT, J. T. 2010. "A silent revolution? New management ideas and the reinvention of European public employment services". Socio-Economic Review 8 (3): 461-486. DOI: https://doi.org/10.1093/ser/mwq004. 